Como citar este artículo: Giraldo-Carmona J, Narváez-Solarte W, Díaz-López E. Probióticos en cerdos: resultados contradictorios. Revista Biosalud 2015; 14(1): 81-90. DOI: 10.17151/biosa.2015.14.1.9

\title{
PROBIÓTICOS EN CERDOS: RESULTADOS CONTRADICTORIOS
}

\author{
John Giraldo-Carmona ${ }^{1}$ \\ William Narváez-Solarte ${ }^{2}$ \\ Elvis Díaz-López ${ }^{3}$
}

\section{RESUMEN}

El uso de antibióticos a niveles subterapéuticos como promotores de crecimiento, generan preocupación a nivel mundial por la posible resistencia de algunos microorganismos a ciertos antibióticos, que podrían de manera potencial transferir genes resistentes desde los animales hacia la microbiota humana; existen alternativas como los probióticos y prebióticos, que estimulan la inmunidad del huésped y no tienen residuos en los productos de origen animal, por lo cual se pretende en esta investigación estudiar sus efectos en la nutrición porcina e identificar las principales falencias que se presentan en la experimentación que se realiza con estos. Los probióticos son microorganismos vivos que cuando se administran en la cantidad adecuada, le generan un efecto benéfico al huésped, disminuyen los problemas de salud y pueden aumentar la productividad, gracias a que con ellos se pueden afectar las proporciones de las diferentes especies de bacterias en la microbiota del tracto gastrointestinal. No obstante, en cuanto a su efecto como promotores de crecimiento los resultados son contradictorios, en gran medida por la diversidad de cepas, especies de microorganismos, dosis, la forma de administración; así como también la diferente composición de las dietas utilizadas en los bioensayos.

Palabras clave: ácido lácticas, gastrointestinal, microbiota, porcinos, rendimiento.

\section{PROBIOTICS IN SWINE: CONTRADICTORY RESULTS}

\section{ABSTRACT}

The use of antibiotics at a sub-therapeutic level as growth promoters raises concern worldwide because of the possible tolerance that some microorganisms can develop against some antibiotics which could, in a potential manner transfer resistance genes from animals to human microbiota. There are some alternatives such as probiotics and prebiotics that stimulate the host's immunity and do not leave any residue in any animal origin products; therefore, this research intends to study its effects on the pigs' diet and to identify the main deficiencies visible in the experimentation carried out with them. Probiotics are live microorganisms that, when administered in the right amount, create benefic effects in the host, lowering health problems, and raising productivity because they can affect the proportions of different species of bacteria in the in the gastrointestinal tract microbiota; however, as far as its effect as growth promoters, the results are contradictory mainly because of the wide diversity of strains and species doses and administration forms as well as different composition of the diets used in the bioassays.

Key words: gastrointestinal, lactic acid, microbiota, performance, swine.

\footnotetext{
${ }_{1}^{1}$ Médico Veterinario Zootecnista, Estudiante maestría Ciencias Veterinarias, Universidad de Caldas. Manizales, Colombia. jhonalejandro88@hotmail.com

2 Doctor, Departamento de Salud Animal, Universidad de Caldas, Grupo de Investigación en Nutrición, Metabolismo y Seguridad Alimentaria. Manizales, Colombia.wnarvaez@ucaldas.edu.co

${ }^{3}$ M.Sc., Departamento de Producción Agropecuaria, Universidad de Caldas. Manizales, Colombia. elvis.diaz@ucaldas. edu.co
} 


\section{INTRODUCCIÓN}

La necesidad de mejorar las empresas pecuarias, disminuyendo los costos de producción y maximizando la productividad, llevaron al uso de antibióticos a niveles subterapéuticos como promotores de crecimiento, con el propósito de disminuir la incidencia de algunas enfermedades y mejorar la utilización de los nutrientes por parte del animal; sin embargo, estas sustancias generaron una preocupación a nivel mundial por la posible resistencia de algunos microorganismos a ciertos antibióticos, y que podrían de manera potencial transferir genes resistentes desde los animales hacia la microbiota humana (1), lo cual llevó a su prohibición por la Unión Europea desde el año 2006 (2).

La búsqueda de nuevas alternativas a los antibióticos promotores de crecimiento se incrementó, y actualmente se buscan compuestos que aumenten la inmunidad del huésped y no tengan efectos secundarios o residuales en los productos de origen animal (3); es allí donde los probióticos, prebióticos y simbióticos tienen un gran potencial por su efecto modulador de la microbiota del tracto gastrointestinal, que genera efectos positivos en el huésped.

En cuanto al uso de probióticos en la producción porcina, existen problemas metodológicos en los bioensayos que impiden concluir de manera veraz su efectividad como promotores de crecimiento, es por esto que en esta investigación de tipo documental se pretende estudiar los efectos de los probióticos en la alimentación porcina y algunas falencias que se presentan en los experimentos que evalúan estas sustancias.

\section{MICROBIOTA INTESTINAL}

La microbiota del tracto gastrointestinal en mamíferos es compleja y biodiversa, posee una gran cantidad de especies y células que superan las $10^{14}(4-6)$, es altamente activa e interactúa de manera directa con el huésped (7).
Las bacterias intestinales se dividen en especies que producen efectos perjudiciales como: diarrea, infección, daño hepático, carcinogénesis y putrefacción intestinal; y especies que generan efectos benéficos como: estimulación del sistema inmune de manera no inflamatoria, disminución de los problemas de distención por gases, mejora en la digestión y absorción de nutrientes, producción de ácidos grasos volátiles y participación en la síntesis de vitaminas, principalmente del complejo B. Cabe señalar que algunos de estos efectos se deben, principalmente, a la inhibición del crecimiento de bacterias perjudiciales $(7,8)$.

\section{PROBIÓTICO}

\section{Definición de probiótico}

Según la FAO/WHO (9) un probiótico es un "microorganismo vivo que cuando se administra en la cantidad adecuada, le genera un efecto benéfico al huésped".

Los probióticos pueden ser útiles en las producciones pecuarias porque mejoran el bienestar de los animales, disminuyen los problemas de salud y, por ende, pueden aumentar la productividad; además de estar acorde con las normas legales y las exigencias para alimentos funcionales bioseguros del consumidor $(10,11)$.

Una de las experiencias de aceptación de estos productos en la dieta humana, ha demostrado que bebidas lácteas con inclusión de Lactobacillus casei solo o en combinación con L. plantarum, L. acidophilus, Bifidobacterium animalis subsp. lactis y dos cepas de Bifidobacterium breve, aumentan la actividad de las células NK y la concentración de inmunoglobulina A salival en niños después de 30 días de consumir los productos, y debido a que esta inmunoglobulina juega un papel central en la inmunidad local, su incremento puede reforzar la resistencia de la mucosa a las infecciones (11). Según los mismos 
autores, la modulación del sistema inmune está estrictamente correlacionada con la presencia de las bacterias probióticas en el intestino.

La capacidad de las cepas exógenas para sobrevivir en el tracto digestivo es considerada un prerrequisito para ejercer su función como probiótico; condición ejemplificada con el comportamiento del la cepa $L$. casei, la cual es influenciada positivamente por la presencia de bifidobacterias en el intestino, reflejándose en la alta viabilidad en heces y en el aumento de la población de lactobacillus y bifidobacterias intestinales, demostrando así buena resistencia de la cepa a las condiciones adversas y su adaptación a los cambios repentinos del ambiente intestinal (11).

Entre las cepas de mayor utilización como probióticos se encuentran principalmente bacterias Gram positivas como el Lactobacillus, el Enterococcus, el Bacillus, el Bifidobacterium y la Sacharomyces $(7,12)$.

En experimentos recientes se han obtenido probióticos de alimentos fermentados, que en su gran mayoría son microorganismos mesófilos, aunque también se pueden encontrar algunas arqueobacterias extremófilas de las cuales aún no se conoce con exactitud cómo sus metabolitos tienen efectos probióticos en los mamíferos (13). Los efectos benéficos de los probióticos son específicos de cada cepa bacteriana, por tal motivo, siempre primero se debe hacer la identificación de las cepas para relacionarlo con un efecto específico en la salud, así como también para permitir la vigilancia y posteriores estudios epidemiológicos, lo cual implica que cada cepa o combinación de cepas deben ser probadas y evaluadas para conocer su efecto benéfico $(9,14)$.

Fuller (15) describe cuatro formas posibles de cómo funcionan los probióticos en el tracto gastrointestinal. La primera es la producción de sustancias antimicrobiales: estas sustancias pueden reducir el número de células viables, interferir en su metabolismo o en la producción de toxinas; la producción de ácidos grasos volátiles, principalmente el ácido láctico por parte de las bacterias ácido lácticas reducen la colonización del intestino por parte de las bacterias patógenas. Segunda, competencia por receptores de adhesión: algunos probióticos se adhieren a la pared intestinal y por lo tanto compiten por los sitios de adhesión. Tercera, competencia por nutrientes: aunque el intestino es muy rico en nutrientes y es poco probable que se compita por nutrientes, la disminución de solo un nutriente puede influir en la composición de la microbiota. Cuarta, estimulación de la inmunidad: se ha encontrado que los lactobacillus estimulan la actividad de los macrófagos contra diferentes especies de bacterias ya sea por absorción de antígenos específicos o translocación del lactobacilo al torrente sanguíneo.

\section{Efecto de los probióticos sobre la microbiota y el tracto gastrointestinal en cerdos}

Las bacterias probióticas son transferidas desde la cerda a los lechones por contacto con las heces maternas, antes de que éstos inicien el consumo de alimento sólido (16). En el periodo del destete se genera una disrupción en la microbiota normal del tracto gastrointestinal con cambios en la flora bacteriana del ciego, aumento en las enterobacterias y disminución de las bacterias ácido lácticas que abundan en el lechón lactante. Simultáneamente, suceden cambios en la histología del ciego, aumentándose la actividad proliferativa de las criptas y del sistema inmune en la mucosa cecal. Castillo et al. (17) observaron que el peso del ciego de lechones destetados que consumen alimentos balanceados es mayor que el de aquellos que a la misma edad solo consumen leche, y sostienen que este efecto se debe al incremento del material fermentable proveniente de la dieta, que da inicio a la actividad fermentativa a nivel de colon.

Lahteinen et al. (18) afirman que muchas cepas de Bacillus pueden ser aisladas del tracto 
gastrointestinal de los cerdos y ser usadas como probióticos promisorios, gracias a que tienen buena tolerancia al ácido gástrico y al jugo biliar. También manifiestan que en sus investigaciones no han encontrado relación entre el sitio del intestino de donde se aísla la cepa y su potencial actividad probiótica.

En los estudios revisados, la morfología intestinal, la longitud de las vellosidades y la profundidad de las criptas no se vieron afectadas por el uso de diferentes especies de probióticos $(19,20)$, mientras tanto Ross et al. (10) concluyeron que las estructuras morfológicas de animales alimentados con probióticos mejoran (Tabla 1). Cabe resaltar que la totalidad de las investigaciones dan cuenta de que existe modificación en la microbiota del tracto gastrointestinal, mostrando aumento en el recuento de las bacterias ácido lácticas, que tienen mayor relación con el probiótico suministrado, y disminución en el recuento de bacterias patógenas como E. coli, así como el de los coliformes fecales $(10,14,21,22)$; generándose un efecto mayor, a medida que se aumenta el tiempo de suministro del probiótico (23).

Taras et al. (16) observaron que las heces provenientes de lechones que recibieron en la dieta probióticos presentaron mejor consistencia; y que la incidencia de diarreas postdestete en estos animales se vio disminuida (14).

\section{Efecto de los probióticos sobre el rendimiento zootécnico}

Numerosos estudios se han realizado en lechones alimentándolos con diferentes cepas de bacterias con el fin de evaluar su efecto probiótico (Tabla 1 ), ya sea suministradas en el alimento, en el agua de bebida o directamente al animal vía oral; de la misma forma se han evaluado preparados de bacterias, que combinan diferentes cepas para potencializar el efecto probiótico y así mejorar el rendimiento de los animales, o usando un solo tipo de bacteria. Al analizar los diversos resultados obtenidos se observa discrepancia entre ellos, mientras algunos autores encuentran superior rendimiento zootécnico de los animales que consumen los probióticos, como aumento del consumo de alimento y la ganancia de peso, sin efecto sobre la conversión alimenticia (23), otros como Taras et al. (16) y Mallo et al. (21) encontraron aumento en la eficiencia alimenticia de un $8 \%$ y mayor eficiencia en la conversión alimenticia, pasando esta de 1,74 a 1,60 , respectivamente; por su parte, Guerra et al. (23) además del incremento en la ganancia y la mejoría en la conversión alimenticia, observaron disminución en el consumo de alimento; disminución también observada por Ross et al. (10), aunque ellos no encontraron efecto de los probióticos sobre la ganancia de peso. Giang et al. (14) encontraron en los lechones alimentados con probióticos mejora en las tres variables de rendimiento zootécnico, como son ganancia de peso, consumo de alimento y conversión alimenticia. No obstante estos resultados, son varias las investigaciones en las cuales se manifiesta que la adición de probióticos en la dieta de los lechones no ocasiona ningún tipo de mejora en los parámetros zootécnicos (20, 23-25).

También se han utilizado probióticos en cerdas gestantes para determinar si producen una mejora en la cantidad y calidad de las camadas, así como la reducción de mortinatos (Tabla 2 ), sin embargo nuevamente los resultados no son concluyentes, aun en investigaciones realizadas dentro de un mismo grupo de investigadores, son los casos de Taras et al. (16, 22), quienes encontraron una mejora significativa en el desempeño de las cerdas, mientras que los mismos autores en otros bioensayos no encontraron diferencias entre el grupo de cerdas tratadas y el control (24).

En la primera investigación donde se suministró oralmente un extracto de compost fermentadotermófilo en porcinos, en donde las bacterias más representativas fueron Bacillus thermoamylovorans y Bacillus thermocloacae (26), bacterias poco utilizadas y evaluadas en las investigaciones, se 
redujo el número de mortinatos y se generó un efecto promotor de crecimiento en los lechones, bajo el argumento de que el estado de salud de la madre se ve reflejado directamente en el normal crecimiento de los fetos y los neonatos; aunque todas las dietas experimentales contenían otras bacterias probióticas que pudieron interactuar con las bacterias del extracto, potencializando el efecto o enmascarando algunos efectos que ya se habían generado por las demás bacterias, los autores concluyeron que las bacterias que se forman en el proceso de compostaje termofílico pueden ser una fuente potencial de bacterias probióticas para mamíferos (13).

Aunque Ehrman et al. (27) recomiendan que en vez de utilizar un solo microorganismo se deben utilizar múltiples cepas o mezcla de ellos para aumentar la efectividad del probiótico, respaldado con los resultados obtenidos por Giang et al. (14), existen diversas investigaciones en las que se observa que los preparados con diferentes cepas no mejoraron la productividad de los animales $(20,25)$.

Si bien se podría pensar que el efecto promotor de crecimiento depende de la cepa probiótica utilizada, de la dosis utilizada y del tiempo en el cual fue suministrada, también es de vital importancia tener en cuenta los ingredientes que componen la dieta, ya que el uso de dietas heterogéneas en cuanto a su contenido de ingredientes entre las diversas investigaciones, no permite realizar comparaciones acertadas entre los efectos que tiene una cepa en particular, aunado a esto, en algunas investigaciones no se presenta la composición de la dieta, o utilizan de dietas comerciales que pueden tener una gran variedad de aditivos que interfieran con los resultados obtenidos. Dentro de este contexto, si se comparan el arroz, maíz, trigo y cebada, que son las fuentes energéticas de mayor uso en las dietas experimentales (tablas 1 y 2), se observa que difieren ampliamente en cuanto a su contenido de oligosacáridos y polisacáridos no amiláceos, aumentándose en el mismo orden en el cual se encuentran listados (28); carbohidratos que generalmente se encuentran como constituyentes de la fracción fibrosa del ingrediente y que no pueden ser digeridos por las enzimas digestivas, en cambio, sí son utilizados en gran medida por la microbiota del tracto gastrointestinal, principalmente a nivel de colon y ciego, como sustrato, generando un efecto prebiótico (29) el cual puede enmascarar el efecto de los probióticos adicionados en las dietas. 


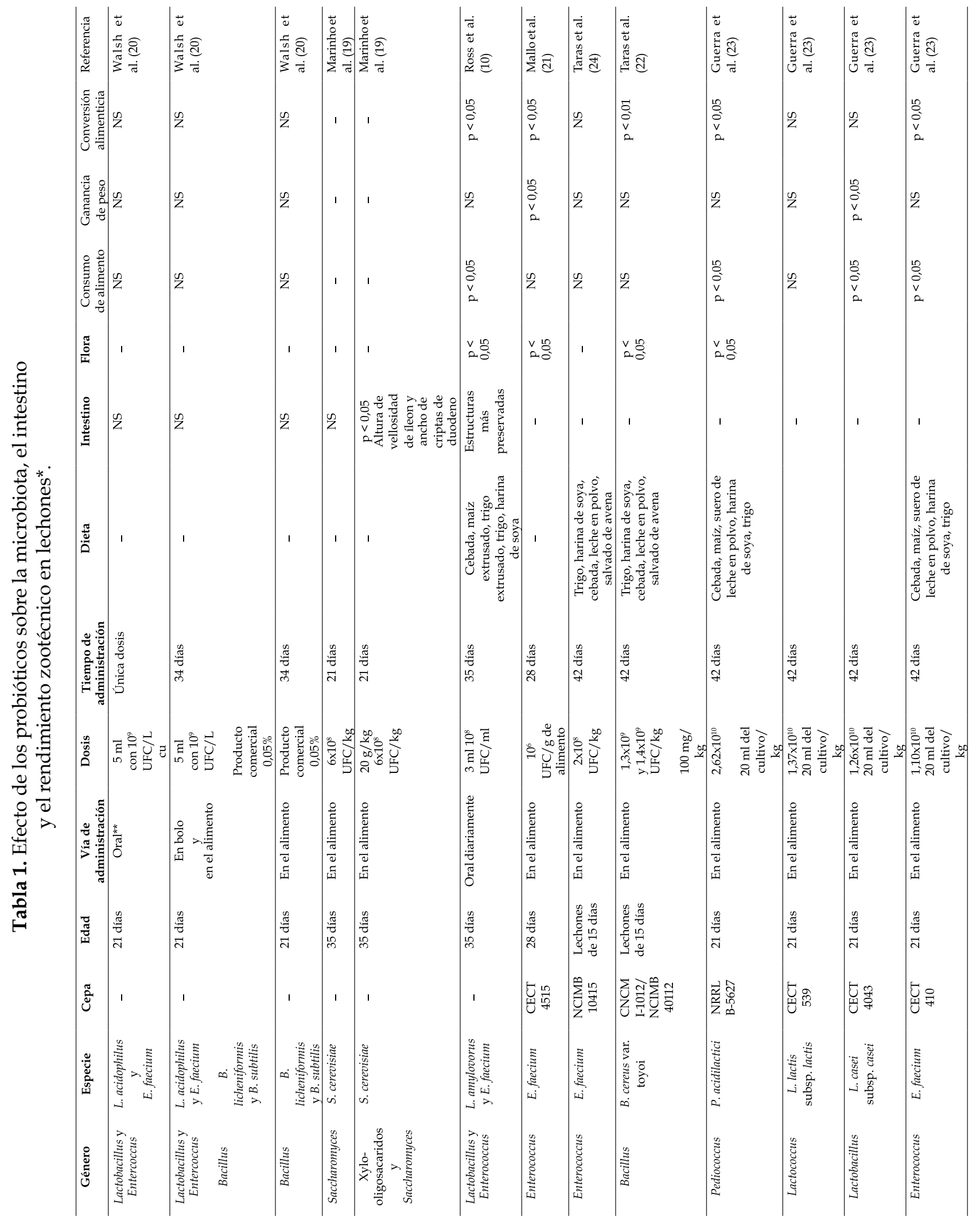




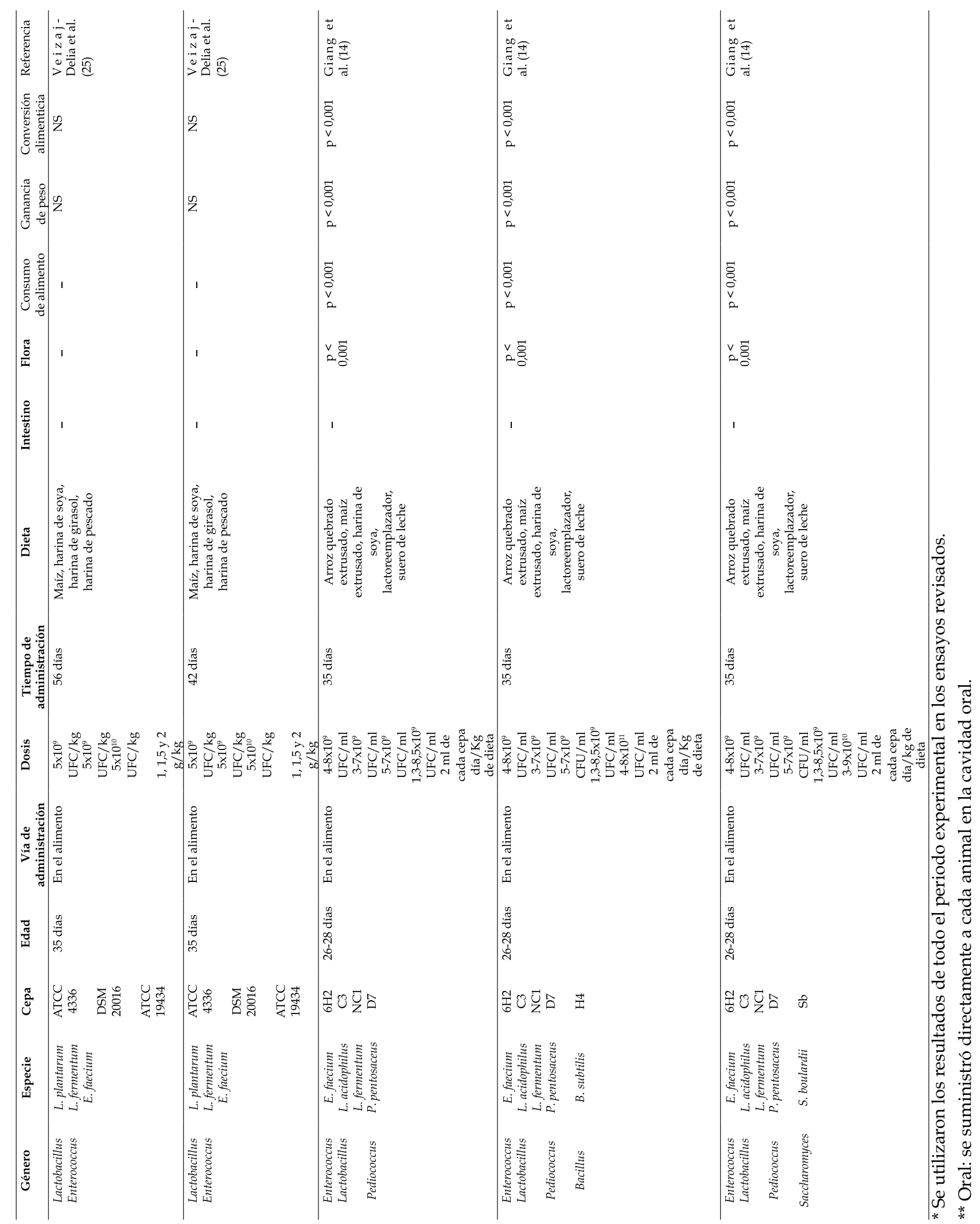




\section{Biosalud}

John Giraldo-Carmona, William Narváez-Solarte, Elvis Díaz-López

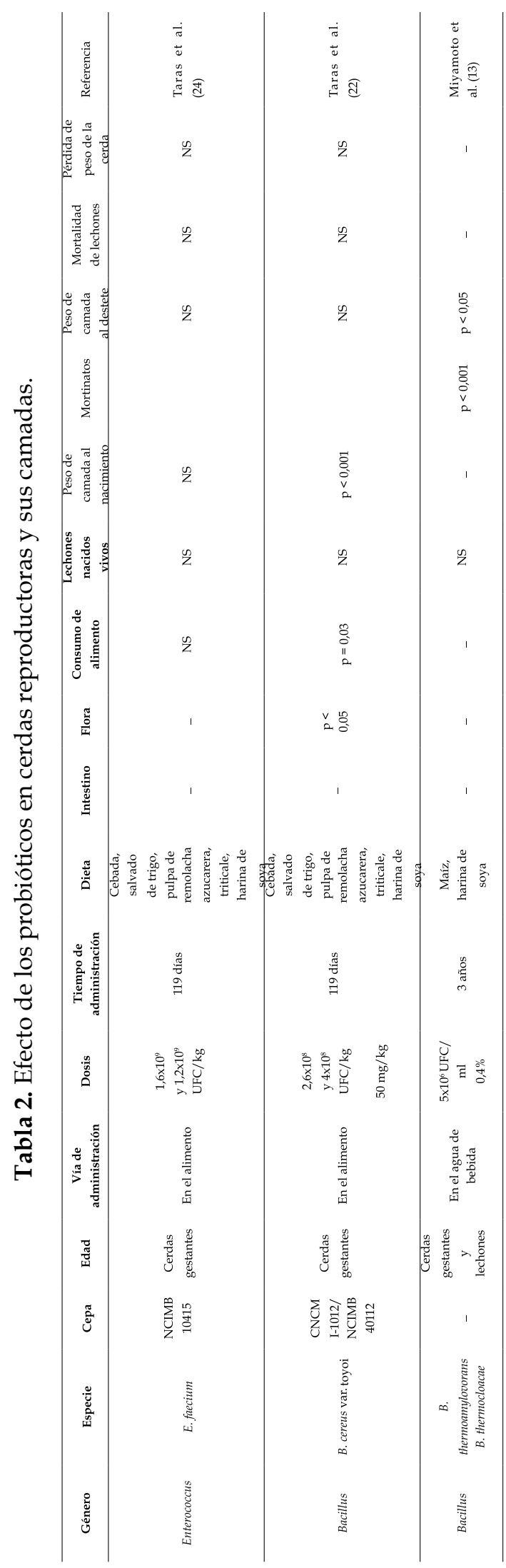




\section{CONCLUSIONES}

La administración de probióticos en la dosis adecuada sin lugar a dudas afecta la composición de la microbiota intestinal de manera benéfica para el huésped, sin embargo en cuanto a su efecto promotor de crecimiento los resultados son contradictorios, en gran medida esto se puede explicar por la gran diversidad de cepas y especies utilizadas a diferentes dosis y formas de administración. La diferente composición de las dietas experimentales puede estimular de forma distinta y en diferentes niveles especies de bacterias benéficas, por lo cual se recomienda realizar un consenso de cuáles materias primas utilizadas en las dietas, deben ser las más adecuadas y de menor interferencia en el crecimiento de una cepa bacteriana determinada, para así poder lograr mayor claridad al comparar los resultados provenientes de los diferentes experimentos con probióticos.

En la presente investigación se recomienda utilizar una dieta a base de maíz amarillo y torta de soya, cuando el objetivo del estudio sea el de determinar la efectividad de una cepa específica, o la combinación de cepas, en el rendimiento zootécnico, la inmunidad, la composición de la microbiota y las características intestinales de los porcinos. Otro factor a tener en cuenta al realizar este tipo de investigaciones es el de determinar el estado de salud de los animales usados como unidades experimentales y las cargas de microorganismos medioambientales de cada explotación que pueden modificar el complejo ecosistema que se encuentra en el tracto gastrointestinal.

Se requiere profundizar en la investigación y selección de las cepas bacterianas o microorganismos con potencial de actividad probiótica, junto con sus sustratos o prebióticos que maximicen su acción, para de esta manera formar compuestos simbióticos que mejoren el bienestar de los animales, promuevan mejor desempeño con menores índices de enfermedad y una menor utilización de antibióticos terapéuticos.

\section{REFERENCIAS}

1. Mathur S, Singh R. Antibiotic resistance in food lactic acid bacteria - a review. International Journal of Food Microbiology 2005; 105:281-295.

2. EC. Commission of the European Communities, Commission Regulation (EC) No.1831/2003. Official Journal of European Union 2003; L268:29-43.

3. Casewell M, Friis C, Marco E, McMullin P, Phillips I. The European ban on growth-promoting antibiotics and emerging consequences for humans and animals health. Journal of Antimicrobial Chemotherapy 2003; 52:159-161.

4. Murphy WJ, Larkin DM, Van der Wind AE, Bourque G, Tesler G, et al. Dynamics of mammalian chromosome evolution inferred from multispecies comparative maps. Science 2009; 309:613-618.

5. Ley RE, Peterson DA, Gordon JI. Ecological and Evolutionary Forces Shaping Microbial Diversity in the Human Intestine. Cell 2006; 124:837-848.

6. Richards JD, Gong J, De Lange CFM. The gastrointestinal microbiota and its role in monogastric nutrition and health with an emphasis on pigs: Current understanding, possible modulations, and new technologies for ecological studies. J. Anim. Sci 2005; 85:421-435.

7. Gaggia $F$, Mattarelli $P$, Biavati B. Probiotics and prebiotics in animal feeding for safe food production. International Journal of Food Microbiology 2010; 141:S15-S28.

8. Gibson GR, Roberfroid MB. Dietary Modulation of the Human Colonie Microbiota: Introducing the Concept of Prebiotics. Journal of Nutrition 1995; 125:1401-1412.

9. FAO/WHO (Food and Agriculture Organization/World Health Organization). Working Group Report on Drafting Guidelines for the Evaluation of Probiotics in Food. Guidelines for the Evaluation of Probiotics in foods. London, Ontario, Canada: s.n.; 2002. p. 1-11. 
10. Ross GR, Gusils C, Oliszewski R, Colombo-de-Holgado S, González SN. Effects of probiotic administration in swine. Journal of Bioscience and Bioengineering 2010; 109:545-549.

11. Zaninia K, Marzottoa M, Castellazzi A, Borsari A, Dellaglio F, Torriani S. The effects of fermented milks with simple and complex probiotic mixtures on the intestinal microbiota and immune response of healthy adults and children. International Dairy Journal 2007; 17:1332-1343.

12. Anadón A, Martínez-Larrañaga MR, Aranzazu-Martínez M. Probiotics for animal nutrition in the European Union. Regulation and safety assessment. Regulatory Toxicology and Pharmacology 2006; 45:91-95.

13. Miyamoto $H$, Kodama $H$, Udagawa $M$, Mori $K$, Matsumoto J, Oosaki $H$, et al. The oral administration of thermophile-fermented compost extract and its influence on stillbirths and growth rate of preweaning piglets. Research in Veterinary Science 2012; 93:137-142.

14. Giang HH, Viet TQ, Ogle B, Lindberg JE. Growth performance, digestibility, gut environment and health status in weaned piglets fed a diet supplemented with a complex of lactic acid bacteria alone or in combination with Bacillus subtilis and Saccharomyces boulardii. Livestock Science 2012; 143:132-141.

15. Fuller R. Probiotics in human medicine. Gut 1991; 32:439-442.

16. Taras D, Vahjen W, Simon O. Probiotics in pigs - modulation of their intestinal distribution and of their impact on health and performance. Livestock Science 2007; 108:229-231.

17. Castillo M, Martín-Orúe SM, Nofrarías M, Manzanilla EG, Gasa J. Changes in caecal microbiota and mucosal. Veterinary Microbiology 2007; 124:239-247.

18. Lahteinen T, Malinen E, Koort JMK, Mertaniemi-Hannus U, Hankimo T, Karikoski N, et al. Probiotic properties of Lactobacillus isolates originating from porcine intestine and feces. Anaerobe 2010; 16:293-300.

19. Marinho MC, Pinho MA, Mascarenhas RD, Silva FC, Lordelo MM, et al. Effect of prebiotic or probiotic supplementation and ileo rectal anastomosis on intestinal morphology of weaned piglets. Livestock Science 2007; 108:240-243.

20. Walsh MC, Saddoris KL, Sholly DM, Hinson RB, Sutton AL, Applegate TJ, et al. The effects of direct fed microbials delivered through the feed and/or in a bolus at weaning on growth performance and gut health. Livestock Science 2007; 108:254-257.

21. Mallo JJ, Rioperez J, Honrubia P. The addition of Enterococcus faecium to diet improves piglet's intestinal microbiota and performance. Livestock Science 2010; 133:176-178.

22. Taras D, Vahjen W, Macha M, Simon O. Response of performance characteristics and fecal consistency to long-lasting dietary supplementation with the probiotic strain Bacillus cereus var. toyoi to sows and piglets. Archives of Animal Nutrition 2005; 59:405-417.

23. Guerra NP, Bernárdez PF, Méndez J, Cachaldora P, Castro LP. Production of four potentially probiotic lactic acid bacteria and their evaluation as feed additives for weaned piglets. Animal Feed Science and Technology 2007; 134:89-107.

24. Taras D, Vahjen W, Macha M, Simon O. Performance, diarrhea incidence, and occurrence of Escherichia coli virulence genes during long-term administration of a probiotic Enterococcus faecium strain to sows and piglets. Journal of Animal Science 2006; 84:608-617.

25. Veizaj-Delia E, Piub T, Lekaj P, Tafaj M. Using combined probiotic to improve growth performance of weaned. Livestock Science 2010; 134:249-251.

26. Niisawa C, Oka S, Kodama H, Hirai M, Kumagai $Y$, Mori K, et al. Microbial analysis of composted product of marine animal resources and isolation of antagonistic bacteria to plant pathogen from the compost. The Journal of General and Applied Microbiology 2008; 54:149-158.

27. Ehrmann MA, Kurzak P, Baver J, Vogel RF. Characterization of lactobacilli towards their use as probiotic adjuncts in poultry. J Appl Microbiol 2002; 33:966-975.

28. Miled IB. Evaluación de complejos enzimaticos en la mejora del valor nutritivo de cereales y leguminosas en la alimentación de pollos en crecimiento. Bellaterra: Universidad Autónoma de Barcelona; 2001.

29. Hogberg A, Lindberg JE. The effect of level and type of cereal non-starch polysaccharides on the performance, nutrient utilization and gut environment of pigs around weaning. Animal Feed Science and Technology 2006; 127:200-219. 